\title{
Semiconductor Quantum Well Lasers With a Temperature-Insensitive Threshold Current
}

\author{
Alfred R. Adams, Life Fellow, IEEE, Igor Pavlovich Marko, Jayanta Mukherjee, Vlad Stolojan, \\ Stephen John Sweeney, Senior Member, IEEE, Agnieszka Gocalinska, Emanuele Pelucchi, \\ Kevin Thomas, and Brian Corbett
}

\begin{abstract}
This paper proposes and demonstrates a new multiquantum well (MQW) laser structure with a temperatureinsensitive threshold current and output power. Normally, the mechanisms that cause the threshold current $\left(I_{\mathrm{th}}\right)$ of semiconductor lasers to increase with increasing temperature $T$ (thermal broadening of the gain spectrum, thermally activated carrier escape, Auger recombination, and intervalence band absorption) act together to cause $I_{\mathrm{th}}$ to increase as $T$ increases. However, in the design presented here, carriers thermally released from some of the QWs are fed to the other QWs so that these mechanisms compensate rather than augment one another. The idea is in principle applicable to a range of materials systems, structures, and operating wavelengths. We have demonstrated the effect for the first time in $1.5 \mu \mathrm{m}$ GaInAsP/InP Fabry-Perot cavity edge-emitting lasers. The results showed that it is possible to keep the threshold current constant over a temperature range of about $100 \mathrm{~K}$ and that the absolute temperature over which the plateau occurred could be adjusted easily by redesigning the quantum wells and the barriers between them. TEM studies of the structures combined with measurements of the electroluminescent intensities from the wells are presented and explain well the observed effects.
\end{abstract}

Index Terms-Diode lasers, quantum well, temperature dependence, thermal stability.

\section{INTRODUCTION}

Q UANTUM well (QW) lasers have been extremely successful in a wide range of applications, with optical fibre communications being of particular importance. However, in spite of their success, their performance is not ideal [1], [2]. For example, the threshold current of semiconductor lasers is often very sensitive to temperature due to various processes depending on the operating wavelength [1]-[10]. As a result, around

Manuscript received January 30, 2015; revised March 11, 2015; accepted March 11, 2015. This work was supported by the EPSRC Impact Acceleration project, EPSRC project EP/H005587/1 (details of the data and how to request access are available from the University of Surrey publications repository at http://epubs.surrey.ac.uk/807588/), in part by the Irish Higher Education Authority Program for Research in Third-Level Institutions (2007-2011) via the INSPIRE program, and by the Science Foundation Ireland under the Irish Photonic Integration Center Award 12/RC/2276 and Grant 10/IN.1/I3000.

A. R. Adams, I. P. Marko, J. Mukherjee, V Stolojan, and S. J. Sweeney are with the Advanced Technology Institute and the Department of Physics, University of Surrey, Guildford, Surrey GU2 7XH, U.K. (e-mail: alf.adams@ @urrey.ac.uk; i.marko@surrey.ac.uk; J.Mukherjee@surrey.ac.uk; v.stolojan@surrey.ac.uk; s.sweeney@surrey.ac.uk).

A. Gocalinska, E. Pelucchi, K. Thomas, and B. Corbett are with the Tyndall National Institute, University College Cork, Cork, Ireland (e-mail: agnieszka.gocalinska@tyndall.ie; emanuele.pelucchi@tyndall.ie; kevin.thomas@ tyndall.ie; brian.corbett@ tyndall.ie).

Color versions of one or more of the figures in this paper are available online at http://ieeexplore.ieee.org.

Digital Object Identifier 10.1109/JSTQE.2015.2413403
$80 \%$ of electrical power is wasted in a $1.55 \mu \mathrm{m}$ InGaAsP laser chip as heat [8]. This has led to the need for thermoelectric coolers and associated control electronics to stabilize the laser temperature. However, such coolers often consume more energy than the laser they are controlling and also add to the overall heat dissipation of the system. Such coolers also tend to have far less long-term reliability than the laser diode itself. There are consequently many circumstances where it would be advantageous and far cheaper to simply compensate for temperature variations by mechanisms built into the epitaxial structure of the laser chip itself. This paper focuses on a new design and demonstration of a MQW laser structure which can overcome the intrinsic temperature sensitivity of the threshold current of QW lasers.

There are several mechanisms that may cause the threshold current $\left(I_{\mathrm{th}}\right)$ of semiconductor lasers to increase rapidly with increasing temperature, $T$ [1], [2]. These include thermal broadening of the gain spectrum leading to increase in the radiative current with increasing $T$ [1], thermally activated carrier escape from the QWs [4], [5], Auger recombination [1]-[8], [11] and inter-valence band absorption [1], [7], [12], [13]. Normally all of these processes act together to cause $I_{\mathrm{th}}$ to increase as $T$ increases. To compensate for the temperature dependence of GaInAs MQW lasers, approaches involving in-built stress have been attempted (characteristic temperature of $I_{\mathrm{th}}, T_{0}$, was improved from 51 to $133 \mathrm{~K}$ at $293 \mathrm{~K}<T<343 \mathrm{~K}$ ) [16], [17] although these have required significant changes to the standard laser fabrication process. In strongly inhomogeneous systems like self-assembled quantum dot lasers improved carrier thermalisation with increasing temperature can partly compensate for the processes which increase $I_{\text {th }}$ leading to a temperature stable operation over a small temperature range [5], [6], [14], [15]. Here we present a novel design of QW active region [18] such that carriers released from some of the QWs are fed to the other QWs in such a way that these mechanisms compensate rather than augment one another. The idea is in principle applicable to a range of materials systems, structures and operating wavelengths. However, we chose to demonstrate the effect for the first time in $1.5 \mu \mathrm{m}$ GaInAsP/InP edge emitting lasers, since these have been so extensively characterized and their parameters are relatively well known [1], [7].

\section{Design AND Simulation}

The design concept can be most easily understood by considering Fig. 1, which shows the variation of the total bandgap with position across the structure. QWs 4, 5 and 6 (W4, W5 and W6 in Fig. 1) provide effectively the active region of a 


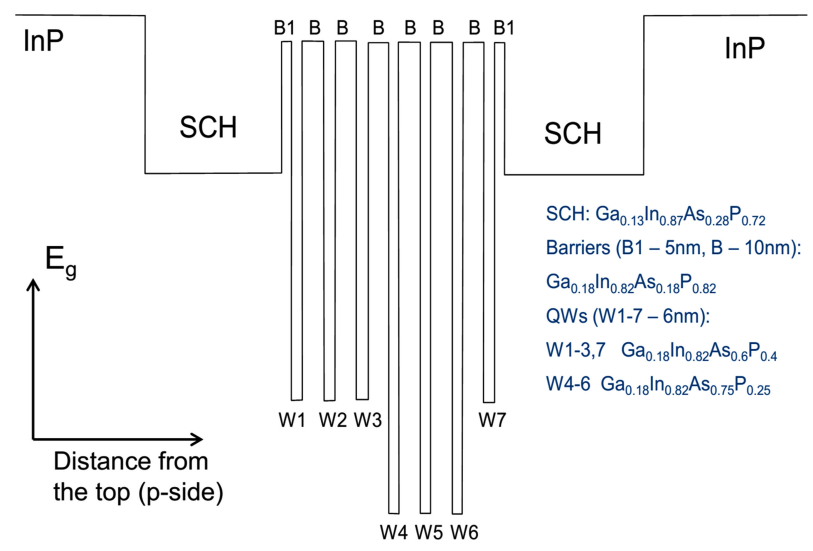

Fig. 1 Schematic diagram of the total bandgap in the designed structure.

compressively strained three-QW $1.5 \mu$ m laser. QWs W $1-3$ and W7 provide carrier traps which, being shallower than the active QWs, release their carriers by thermal excitation as the temperature increases. These released carriers can now transfer to the deeper active QWs and so provide the extra current needed as the non-radiative loss mechanisms increase with increasing temperature. As a result, the total current through the laser can be made to remain approximately constant as the temperature changes. The exact range of temperature over which this occurs depends on the number, depth and distribution of the shallower wells and also on the heights of the barriers between the wells. These are all adjustable parameters in the structure design.

With such a wide variety of parameters available to provide the required effect it was necessary first to investigate the structure theoretically. This was undertaken using LaserMOD (a Synopsys Rsoft design solution) [19] which predicted that an almost constant value of $I_{\mathrm{th}}$ could be achieved over more than $80 \mathrm{~K}$ in the range around room temperature with the bandgap alignment shown in Fig. 1. Care was also taken to maintain overall strain neutrality across the structure. Also, for simplicity of growth, the $\mathrm{Ga}$ and the In compositions were kept constant throughout the QWs so that all the well depths and barrier heights were determined by the As/P ratio. All the QWs were $6 \mathrm{~nm}$ wide while the barriers between them were $10 \mathrm{~nm}$ wide.

Fig. 2 shows the simulated light-current characteristics for this structure. As can be seen, not only is the threshold current constant, but also the slope efficiency. This is primarily because the total current through the device and hence the hole density remained constant as the temperature increases and so intervalence band absorption [12], the primary cause for the degradation of the slope efficiency, also remains essentially constant. As a result, the output power at a fixed current above threshold also stayed almost constant with increasing $T$ as is shown in the inset in Fig. 2 where it is compared to the results for a standard $1.5 \mu \mathrm{m}$ laser [20].

\section{EXPERIMENTAL APPROACH}

The devices were grown by metalorganic vapor phase epitaxy (MOVPE) at low pressure ( $80 \mathrm{mbar}$ ) in a commercial horizontal reactor with purified $\mathrm{N}_{2}$ as the carrier gas. The precursors were trimethylindium (TMIn), trimethylgallium (TMGa), arsine

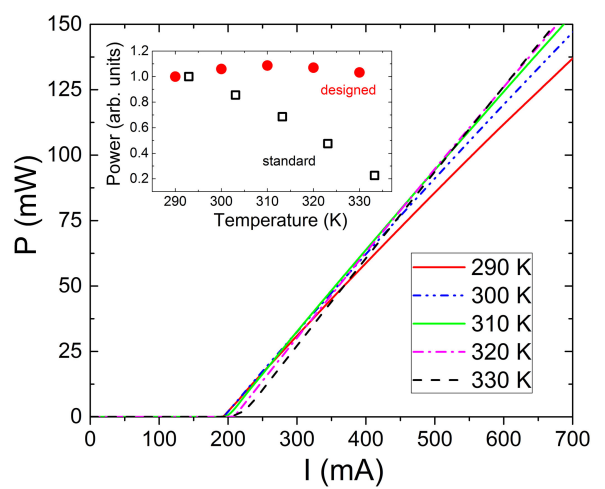

Fig. 2 Temperature dependence of calculated light-output characteristics of the proposed laser. Inset: temperature dependence of the calculated output power (normalized to its value near $293 \mathrm{~K}$ ) in a standard InGaAsP/InP $1.5 \mu \mathrm{m}$ laser (open squares) (see [20, Fig. 37.20]) and in a specially designed laser (full circles) with the same lasing wavelength fabricated according to Fig. 1. The power values were determined at a constant current of $400 \mathrm{~mA}$ for the modelled device.

$\left(\mathrm{AsH}_{3}\right)$, and phosphine $\left(\mathrm{PH}_{3}\right)$, with diethylzinc (DEZn), carbon tetrabromide $\left(\mathrm{CBr}_{4}\right)$ and disilane $\left(\mathrm{Si}_{2} \mathrm{H}_{6}\right)$ used as dopants. Materials were grown on InP ( $\left(\begin{array}{lll}1 & 0 & 0\end{array}\right)$ perfectly oriented n-type substrates. The samples were grown at $630{ }^{\circ} \mathrm{C}$ real estimated growth temperature, with growth rate (Gr) $0.8 \mu \mathrm{m} / \mathrm{h}$ and V/III ratio of 500 for $\mathrm{InP}$ [21] and growth rate $\mathrm{Gr}=0.95 \mu \mathrm{m} / \mathrm{h}$ and V/III ratio from 100 to 350 in case of different compositions of InGaAsP. All samples were characterized as grown for quality control by Nomarski interference contrast microscopy and atomic force microscopy, revealing mirror-like surfaces typical for MOVPE surface step organization. X-Ray diffraction measurements were undertaken to confirm crystallinity and to determine the lattice parameter of the grown layers. Doping levels were estimated by capacitance-voltage measurements. Devices were fabricated with 50 and $80 \mu \mathrm{m}$ wide stripe contacts so that any temperature dependent current spreading was negligible. The devices were produced with as-cleaved facets and 1 and $1.5 \mathrm{~mm}$ cavity lengths.

The devices were mounted in a closed cycle cryostat with temperature control over a temperature interval of 20-300 K. The devices were operated in a pulsed regime $(1 \mathrm{kHz}$ frequency and $500 \mathrm{~ns}$ pulse width) to avoid self-heating effects. The emission from one facet was collected using a standard multimode optical fibre. To compare the temperature behavior of the emission spectra from the shallow and deep QWs, measurements were also carried out at a low continuous wave current of $50 \mathrm{~mA}$ $\left(\sim 35 \mathrm{~A} / \mathrm{cm}^{2}\right)$ where the self-heating effect is negligible. The emission spectra in the cryostat were measured using a fibre coupled optical spectrum analyzer. Due to multimodal lasing operation and therefore variation in the collection efficiency of the light by the fibre, such arrangements are not suitable for accurate slope efficiency measurements. Therefore only the variation in threshold current could be reliably monitored in these measurements as presented in this paper.

\section{RESUlTS AND DISCUSSION}

The temperature dependencies of the threshold current in the fabricated devices are shown in Fig. 3. Sample "A" was the first 


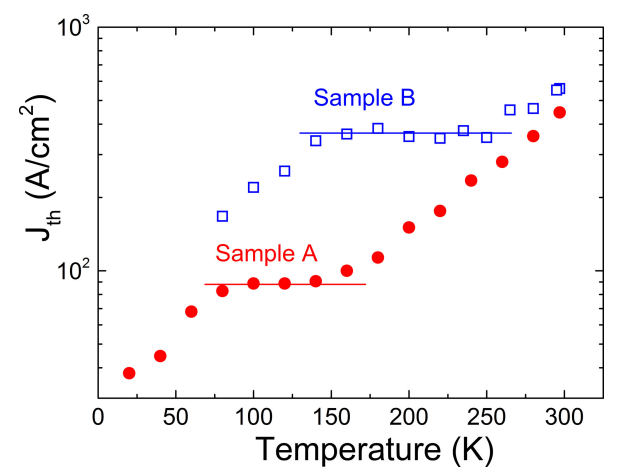

Fig. 3. Temperature dependence of threshold current density in two InGaAsP/InP laser structures grown according to a simulated design with predicted temperature insensitive behaviour.

to be grown and as can be seen, it displayed a constant threshold current over a range of approximately $70 \mathrm{~K}$ but centered at a lower temperature (80-150 K) than predicted by the simulation in Fig. 2. The device lased at $1.63 \mu \mathrm{m}$ at room temperature and analysis of the emission spectra showed that the growth differed significantly from the nominal design which may be due to uncertainties in both the modeling parameters and/or growth conditions. However, the fact that a region of constant $I_{\mathrm{th}}$ existed indicated that the concept worked in principle. Taking into account the results for sample "A", the growth parameters were empirically adjusted and sample "B" was grown (QWs 1, 2, 3, 7: $\mathrm{In}_{0.82} \mathrm{Ga}_{0.18} \mathrm{As}_{0.62} \mathrm{P}_{0.38} ; \mathrm{QWs} 4,5,6: \mathrm{In}_{0.82} \mathrm{Ga}_{0.18} \mathrm{As}_{0.68} \mathrm{P}_{0.32}$ ). As can be seen the flat $I_{\mathrm{th}}$ region was successfully moved from low temperature and now spanned from approximately 140 to $270 \mathrm{~K}$. This device lased at $1.47 \mu \mathrm{m}$ at room temperature indicating that the structure was still not exactly as designed. However, the changes made between sample "A" and sample " $B$ " were well within the limits of the variations available and therefore provides confidence that further iterations would make it possible to produce a device able to work at, for example, $1.55 \mu \mathrm{m}$ and with a constant $I_{\text {th }}$ at room temperature and above.

To confirm that the desired carrier transfer processes were indeed occurring, studies were made as a function of temperature of the emission from the end facet at a fixed current below threshold. The spectra observed for the two samples are shown in Fig. 4.

We consider first the results for sample "A". As can be seen, two peaks are clearly visible corresponding to the shallow (peak S) and deep (peak D) QWs. It should be noted that there is no change in the height of peak $\mathrm{S}$ between 20 and $40 \mathrm{~K}$ at which temperatures $I_{\text {th }}$ increases with increasing temperature. However, between 80 and $120 \mathrm{~K}$ there is a clear decrease in peak $\mathrm{S}$ as carriers escape from the shallow wells and over this range there is no change in $I_{\mathrm{th}}$ indicating that the carriers have transferred to the deeper wells. Above $160 \mathrm{~K}$ the magnitude of peak $\mathrm{S}$ decreases to zero, indicating that the shallow QWs had been depleted of carriers and so could no longer supply the deep QWs, so resulting in a resumed increase in $I_{\mathrm{th}}$. Another significant feature is that the photon energy corresponding to peak $\mathrm{S}$ indicates a shallower QW than designed and partly explains why it empties at lower temperatures than intended, causing the

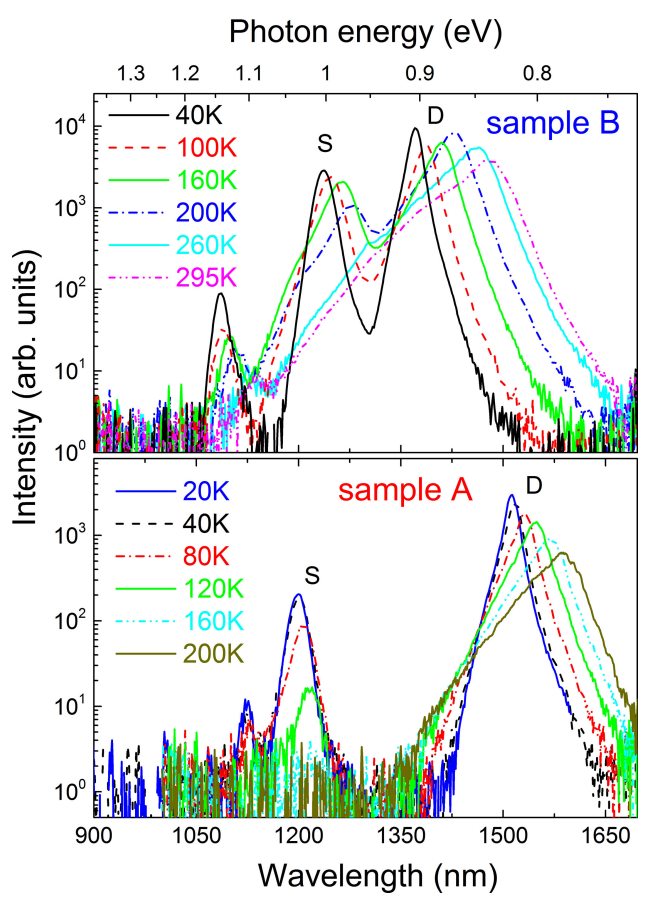

Fig. 4. Emission spectra at a constant low injection current $(50 \mathrm{~mA})$ at different temperatures, demonstrating temperature induced carrier redistribution between the shallow (S) and deep (D) QWs.

low temperature range of the plateau in $I_{\mathrm{th}}$. Similar studies were made on sample "B" and are shown for comparison in Fig. 4. They showed the same effect of the decreasing height of $\mathrm{S}$ over the range of the plateau in $I_{\mathrm{th}}$. However, this had now moved to the temperature range $150 \mathrm{~K}-250 \mathrm{~K}$, supporting the above analysis of sample "A". It should also be noted that peak $\mathrm{S}$ is at longer wavelengths in sample "B" corresponding to deeper wells and hence higher release temperature than in sample "A". While the luminescence spectra only give information about the energy separations of the valence and conduction band edges, the total depth of the wells depends on the barrier heights. Further information about these is provided by the TEM studies below.

\section{FURTHER ANALYSIS}

To clarify that the designed effects were occurring and to help explain why they were not happening over the intended temperature range, transmission electron microscopy of the laser structures was carried out.

Thin film sections for electron microscopy were prepared using an FEI Novalab dual beam focused ion beam microscope, using Ga ions accelerated at $30 \mathrm{keV}$. The beam damage induced by the energetic $\mathrm{Ga}$ ions was reduced with a final stage polish of a few ion beam passes with the foil tilted to $\pm 5^{\circ}$ [22]. However, artefact In islands (as seen in Fig. 5) are expected to form on the surface as a result of ion beam milling [23]. The foils were examined in a Hitachi HD2300A field-emission scanning transmission electron microscope (STEM), operated at $200 \mathrm{keV}$. The STEM has three modes of imaging: Secondary-electron, transmission (bright field) and atomic contrast (high-angle annular dark field, between 80 and $250 \mathrm{mrad}$ scattering semi-angle). 


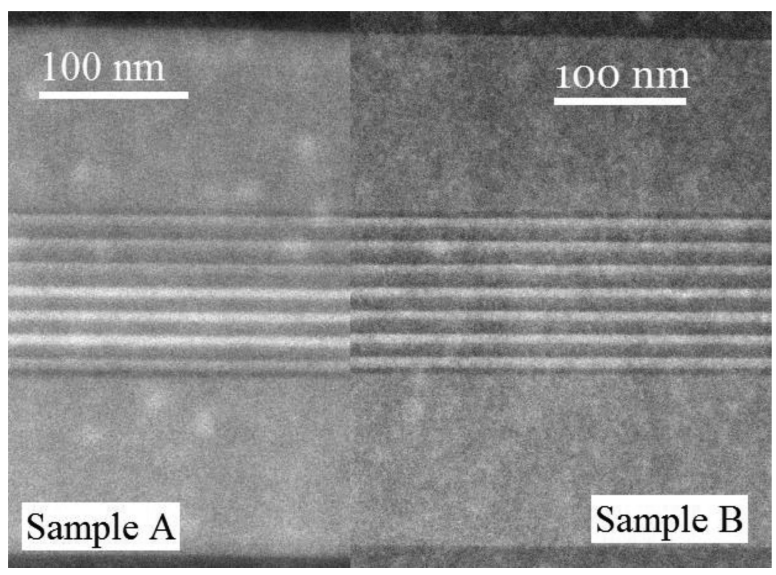

Fig. 5. Z-contrast image of devices "A" and "B", showing three shallow QWs, followed by three deeper QWs (higher As content) and the final QW with the same contrast as the first three.

TABLE I

BARRIER AND QW THICKNESSES

\begin{tabular}{lcccccccc}
\hline \hline Barrier [nm] & 5.1 & 9.8 & 9.7 & 9.8 & 9.8 & 10.7 & 10.0 & 5.0 \\
\hline QW [nm] & 6.1 & 6.1 & 6.0 & 6.3 & 6.0 & 6.0 & 6.0 & \\
\hline \hline
\end{tabular}

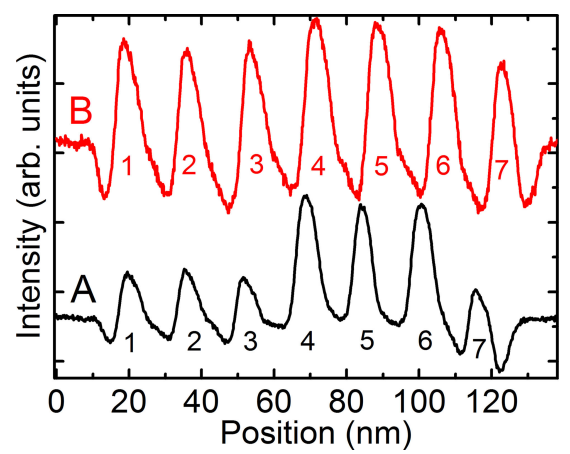

Fig. 6. An integrated Z-contrast intensity profile across the devices "A" and "B" (integrated over $140 \mathrm{~nm}$-wide portion of the device (see Fig. 5).

Z-contrast (where $\mathrm{Z}$ is the atomic number) imaging does not have diffraction contrast, so the intensity is proportional to the thickness, the density of the material and $Z^{2}$. That is, a feature in Z-contrast appears brighter if it is thicker, denser, and much brighter if it has a higher atomic number, which means that for a flat or wedge sample, changes in contrast are directly related to changes in the density and the composition of the material, allowing for some qualitative interpretation of doping profiles.

Fig. 5 shows a higher resolution Z-contrast image of the lasing structures, with the SCH layers measuring $120 \mathrm{~nm}$, with bright spots where In islands form during ion-beam milling. Table I summarises the barrier and QW measured thicknesses, from top to bottom, with $0.2 \mathrm{~nm}$ standard deviation, indicating that they are very close to the design.

Fig. 6 shows diagrammatically the variation of $\mathrm{Z}$, the As/P ratio, across the structure as derived from the results shown in Fig. 5. Because, in general, alloys containing a higher fraction of atoms with a high atomic number have a smaller bandgap, the variation in $\mathrm{Z}$ reflects the inverse of the variation in bandgap.

In other words, Fig. 6 is a reflection of the designed structure shown in Fig. 1. Broadly speaking this appears to be correct. However, several interesting features are apparent. The QWs are numbered 1 to 7 in Fig. 6, 1 being nearest to the surface. We are considering first the results for sample "A." Well 7 appears to have a smaller As concentrations than QWs $1-3$, whilst QWs 4-6 have an increased As concentration, where QW 4 appears slightly higher than QWs 5 and 6. We also note that the barriers between QWs 3-6 do not seem to fall in intensity to the levels of the barriers between QWs 1-3 and 6-7. This indicates that the barriers between these QWs were not as high as intended and this is almost certainly a further reason for the low temperature of the plateau in $I_{\mathrm{th}}$. The large difference between the peaks in the $\mathrm{Z}$ values between the shallow and deep QWs is consistent with the large energy separation between the emission peaks in Fig. 4. Considering now the results for sample " $\mathrm{B}$ " we see first that the $Z$ values (As content) in the shallow wells (1-3 and 7) are much closer to the $Z$ value in the deep wells explaining the longer wavelength peak $S$ in sample B. Secondly, we see that the value of $\mathrm{Z}$ in all the barrier regions falls well below the $\mathrm{Z}$ value in the outlying $\mathrm{SCH}$ regions and designed in Fig. 1. Thus we have higher barriers and so deeper wells so explaining the move of the plateau in $I_{\mathrm{th}}$ to higher range of T than in sample "A".

In summary, information about the variation of well depths and barrier heights obtained from TEM studies when added to the results of the luminescence measurements seem to explain very well the observed plateaus in $I_{\text {th }}$ observed and the change in temperature range between samples "A" and "B". Because there is such a range of further well depths and barrier heights possible from the alloys available, we can be confident that further adjustments can move the plateau to room temperature and above as predicted by the structure shown in Fig. 1 .

\section{CONCLUSION}

We have modelled theoretically and demonstrated experimentally a new design of QW structure in which the threshold current is insensitive to temperature changes over a range of approximately $100 \mathrm{~K}$. The modelling indicates that the output power of these devices would also be constant at a fixed current. Analysis of the emission spectra from the device indicates that the desired transfer of charge is indeed occurring. Also the discrepancies seen in the spontaneous emission peak energies combined with TEM studies, explain qualitatively why these initial devices show the effects at temperatures below room temperature as was intended in the design. Further steps are now planned to extend these preliminary growths to move the plateau in $I_{\text {th }}$ to room temperature and above and to study variations in slope efficiency and output power. The generic design approach we have taken and applied to $1.5 \mu \mathrm{m}$ devices is equally applicable to $1.3 \mu \mathrm{m}$ lasers as well as shorter wavelengths and to mid-infrared devices in both edge-emitting and vertical cavity geometries. What is more, the structures can be grown using standard MOVPE or MBE techniques on the same cost basis as conventional devices. We therefore believe that the new design has wide applicability 
for the simplification of a variety of semiconductor laser based systems leading to significant energy and cost savings.

\section{REFERENCES}

[1] A. F. Phillips, S. J. Sweeney, A. R. Adams, and P. J. A. Thijs, "The temperature dependence of 1.3- and 1.5- $\mu \mathrm{m}$ compressively strained $\operatorname{InGaAs}(\mathrm{P})$ MQW semiconductor lasers," IEEE J. Sel. Topics Quantum Electron., vol. 5, no. 3, pp. 401-412, May/Jun. 1999.

[2] I. P. Marko and S. J. Sweeney, "Optical and electronic processes in semiconductor materials for device applications," in Excitonic and Photonic Processes in Materials (Springer Ser. Mater. Sci.), J. Singh and R. T. Williams, Ed. New York, NY, USA: Springer-Verlag, 2015, vol. 203, pp. 253-297.

[3] R. Fehse et al., "A quantitative study of radiative, auger, and defect related recombination processes in 1.3- $\mu \mathrm{m}$ GaInNAs-based quantum-well lasers," IEEE J. Sel. Topics Quantum Electron., vol. 8, no. 4, pp. 801-810, Jul./Aug. 2002.

[4] K. O'Brien et al., "Recombination processes in midinfrared InGaAsSb diode lasers emitting at $2.37 \mu \mathrm{m}, "$ Appl. Phys. Lett., vol. 89, p. 051104, 2006

[5] I. P. Marko et al., "Recombination and loss mechanism in low-threshold InAs/GaAs $1.3 \mu \mathrm{m}$ quantum-dot lasers," IEEE J. Sel. Topics Quant. Electron., vol. 11, no. 5, pp. 1041-1047, Sep./Oct. 2005.

[6] M. T. Crowley et al., "The importance of recombination via excited states in InAs/GaAs $1.3 \mu \mathrm{m}$ quantum-dot lasers," IEEE J. Sel. Topics Quantum Electron., vol. 15, no. 3, pp. 799-807, Sep. 2009.

[7] S. J. Sweeney and P. J. A. Thijs, "Origin of the high temperature performance degradation of $1.5 \mu \mathrm{m} \mathrm{InGaAs(P)/InP} \mathrm{quantum} \mathrm{well} \mathrm{lasers,"} \mathrm{in}$ Proc. IEEE 16th Аnnu. Meet. Lasers Electro-Optics Soc., 2003, vol. 2, pp. 977-978.

[8] S. J. Sweeney, A. F. Phillips, A. R. Adams, E. P. O'Reilly, and P. J. A. Thijs, "The effect of temperature dependent processes on the performance of $1.5-\mu \mathrm{m}$ compressively strained $\operatorname{InGaAs}(\mathrm{P}) \mathrm{MQW}$ semiconductor diode lasers," IEEE Photon. Tech. Lett., vol. 10, no. 8, pp. 1076-1078, Aug. 1998.

[9] T. R. Chen et al., "Carrier leakage and temperature dependence of InGaAsP lasers," Appl. Phys. Lett., vol. 43, pp. 217-218, 1983.

[10] S. J. Sweeney, G. Knowles, and T. E. Sale, "Evaluating the continuouswave performance of AlGaInP-based red $(667 \mathrm{~nm})$ vertical-cavity surfaceemitting lasers using low-temperature and high pressure techniques," Appl. Phys. Lett., vol. 78, p. 865, 2001.

[11] N. K. Dutta and R. J. Nelson, "Temperature dependence of threshold current of InGaAsP/InP double-heterostructure lasers and Auger recombination," Appl. Phys. Lett., vol. 38, pp. 407-409, 1981.

[12] A. R. Adams, M. Asada, Y. Suematsu, and S. Arai, "The temperature dependence of the efficiency and threshold current of $\operatorname{In}_{1-x} \mathrm{Ga}_{x} \mathrm{As}_{1-\mathrm{y}}$ lasers related to intervalence band absorption," Jpn. J. Appl. Phys., vol. 19 , no. 10, p. $621,1980$.

[13] S. Seki, H. Oohashi, H. Sugiura, T. Hirono, and K. Yokoyama, "Study on the dominant mechanisms for the temperature sensitivity of threshold current in $1.3 \mu \mathrm{m} \mathrm{InP-based} \mathrm{strained-layer} \mathrm{quantum-well} \mathrm{lasers,"} \mathrm{IEEE} \mathrm{J.}$ Quantum Electron., vol. 32, no. 8, pp. 1478-1486, Aug. 1996

[14] I. P. Marko et al., "Carrier transport and recombination in p-doped and intrinsic $1.3 \mu \mathrm{m} \mathrm{InAs/GaAs} \mathrm{quantum} \mathrm{dot} \mathrm{lasers,"} \mathrm{Appl.} \mathrm{Phys.} \mathrm{Lett.,}$ vol. 87, p. 211114,2005

[15] S. S. Mikhrin et al., "High power temperature-insensitive $1.3 \mathrm{mu}$ InAs/InGaAs/GaAs quantum dot lasers," Semiconductor Sci. Technol., vol. 20, no. 5, pp. 340-342, 2005.

[16] D. A. Cohen, M. E. Heimbuch, and L. A. Coldren, "Reduced temperature sensitivity of the wavelength of a diode laser in a stress engineered hydrostatic package," Appl. Phys. Lett., vol. 69, pp. 455-457, 1996

[17] D. A. Cohen and L. A. Coldren, "Temperature compensation of the threshold current, differential efficiency, and refractive index of a GaInAs/InP MQW diode laser mounted on a bimetallic heatsink," Electron. Lett., vol. 32, no. 24, pp. 2245-2247, 1996.

[18] A. R. Adams, "Semiconductor laser," U.S. Patent US8 937 978, Jan. 20, 2015

[19] SYNOPSYS LaserMOD ${ }^{\mathrm{TM}}$ product overview, (2014). [Online]. Available at http://optics.synopsys.com/rsoft/rsoft-active-device-lasermod.html

[20] S. J. Sweeney and A. R. Adams, "Optoelectronic devices and materials," Handbook of Electronic and Photonic Materials, S. Kasap and P. Capper, Eds. New York, NY, USA: Springer-Verlag, 2007, pp. 887-916.
[21] A. Gocalinska, M. Manganaro, E. Pelucchi, and D. D. Vvedensky, "Surface organization of homoepitaxial InP films grown by metalorganic vapor-phase epitaxy," Phys. Rev. B , vol. 86, p. 165307, 2012.

[22] L. Lechner, J. Biskupek, and U. Kaiser, "Improved focused ion beam target preparation of (S)TEM specimen-A method for obtaining ultrathin lamellae," Microsc. Microanal., vol. 18, no. 2, pp. 379-384, 2012.

[23] M. Mehta, D. Reuter, A. Melnikov, A. D. Wieck, and A. Remhof, "Focused ion beam implantation induced site-selective growth of InAs quantum dots," Appl. Phys. Lett., vol. 91, p. 123108, 2007.

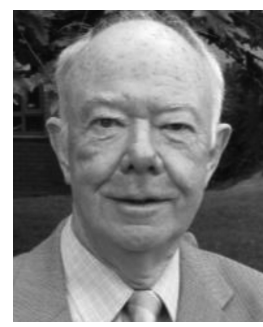

Alfred R. Adams (M'82-SM'00-F'01-LF'06) was born in England, U.K., in 1939. He received the B.Sc., Ph.D., and D.Sc. degrees in physics from the University of Leicester, Leicester, U.K., in 1961, 1964, and 1986, respectively. After completing the Ph.D. research into the electrical and optical properties of orthorhombic sulphur, he spent two years at the University of Karlsruhe, Germany, studying the thermal and thermoelectric properties of selenium and tellurium. In 1967, he joined the University of Surrey, Surrey, U.K., where he is currently a Distinguished Professor of physics, Emeritus. Semiconductor lasers have been one of his major interests since 1980 when he spent sabbatical leave at the Tokyo Institute of Technology, and in 1995, he received the Duddell Medal and Prize by the Institute of Physics, U.K., for proposing intervalence band absorption and for his work on strained layers in lasers. He held the Hitachi Visiting Chair at the Tokyo Institute of Technology in 1992 and was a CNRS Visiting Researcher at the University of Montpellier in 1993. In 1996, he was elected as a Fellow of the Royal Society and he received the Rank Prize for optoelectronics in 2014. He is a Fellow of the Institute of Physics and an Honorary Fellow of the Institute of Engineering and Technology, U.K.

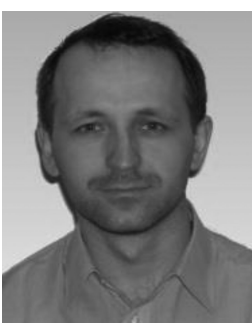

Igor Pavlovich Marko was born in Belarus in 1974 He received the Graduation degree with distinction from the Department of Quantum Radiophysics and Optoelectronics, Belarusian State University, Minsk, Belarus, in 1996, and the Ph.D. degree in 2000. From 1996 until 2001, he studied the optical properties of $\mathrm{ZnSe}$ - and GaN-based wide-bandgap semiconductors and optically pumped lasers with the Laboratory of Semiconductor Optics, B. I. Stepanov Institute of Physics, Belarusian Academy of Sciences. Since 2001, he has been studying the properties of III-Vbased materials and photonic devices and systems from visible to far-infrared spectral range at the University of Surrey.

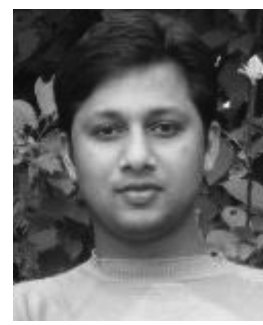

Jayanta Mukherjee was born in India, in 1978, where he received the B.Sc. (Hons.) and M.Sc. degrees in physics, and the M.Tech. degree in laser technology, in 2000, 2002, and 2004, respectively. He then joined the Tyndall National Institute, University College Cork (UCC), Cork, Ireland, where he received the $\mathrm{Ph} . \mathrm{D}$. degree in semiconductor laser physics, in 2009. After eight months of postdoctoral research at the Tyndall Institute, he joined the Advanced Technology Institute, University of Surrey, Surrey, U.K., as a Research Fellow, working on the design and development of laser power converters from 2010 to 2014. He has also worked as an Invited Researcher at the College of Optical Sciences, University of Arizona, Tucson, AZ, USA, and at the Max Born Institute, Berlin, Germany, and as an Assistant Lecturer at UCC, teaching a course on scientific computing from 2007 to 2009 .

He currently holds the Fraunhofer Research Fellowship at the Heinrich Hertz Institute, Berlin, working on power scaling in long-wavelength InP-based lasers. His current research interests include power and brightness scaling in semiconductor lasers, spatio-temporal dynamics in semiconductor lasers, high efficiency photovoltaics, and thermal management of photonic devices. 


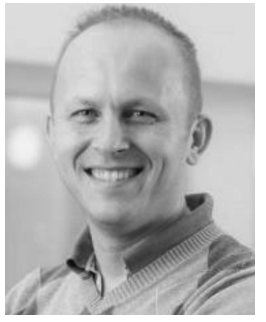

Vlad Stolojan received the B.Sc. degree in physics from the University of East Anglia, Norwich, U.K., in 1996. He received the Ph.D. degree from the University of Cambridge, Cambridge, U.K., becoming an Expert in the application of electron microscopy to study materials on the nanoscale. After a brief postdoctoral research at the University of Cambridge, researching tomographic reconstruction of magnetic fields using electron holography, he moved to the University of Surrey, Surrey, U.K., in 2001 to continue research into electron energy-loss spectroscopy and its applications to nanomaterials characterization. This was followed by a further Postdoctoral Position researching the fundamentals of growth of carbon nanostructures, resulting in an RCUK Fellowship in 2008, converted to a Lectureship in 2011. His current research interests include nanomaterials fabrication and application, particularly carbon nanomaterials, as well as the fabrication of optical microelements for superresolution imaging and electrospinning, for large-area nanomaterials manufacturing. He is a Member of the Institute of Physics and a Fellow of the Royal Microscopical Society.

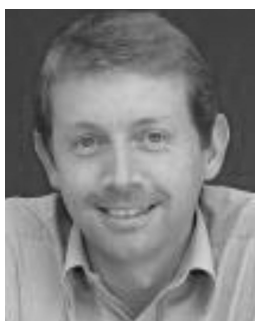

Stephen John Sweeney (S'98-M'99-SM'06) was born in England, U.K., in 1973. He received the B.Sc. (Hons.) degree in applied physics and the Certificate in Education from the University of Bath, Bath, U.K., in 1995, and the Ph.D. degree in semiconductor laser physics from the University of Surrey, Surrey, U.K., in 1999. After two years as a Postdoctoral Research Fellow working on InGaN LEDs and quantum dot lasers, he was the Lead Scientist for Marconi Optical Components on the development of fixed frequency and tunable sources for communications applications. He is currently a Professor of physics at the Advanced Technology Institute, University of Surrey, where he leads the Photonics group. His current research interests include novel quantum well and quantum dot materials operating from the visible through to the midinfrared for applications in solid-state lighting, lasers, detectors and photovoltaics. In addition to his role at Surrey, he is the Chief Technology Officer for ZiNIR Ltd., a U.K. photonics SME, an Associate Editor of the IEEE JOURNAL OF QUANTUM ELECTRONICS, and on the Editorial Board of the Journal of Materials Science: Materials in Electronics. He is a Chartered Physicist and a Fellow of the Institute of Physics, London, U.K

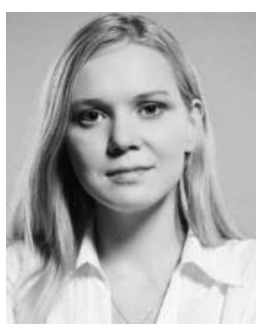

Agnieszka Gocalinska was born in Brzeg, Poland, in 1982. She received the M.Sc. (mgr inż.) degree in applied physics from the Wrocław University of Technology, Wrocław, Poland, in 2005, and the Ph.D. degree in solid-state physics from the University of Cagliari, Cagliari, Italy, in 2010. She was a Marie Curie Fellow in frames of RTN Nanomatch in FP6.

She received the European Reintegration Grant in 2010 to investigate epitaxy of pyramidal QDs. She works within Epitaxy and Physics of Nanostructures Group, Tyndall National Institute, University College Cork, Cork, Ireland, where her main focus is epitaxy of III-V semiconductors for optoelectronic applications and quantum dots growth.

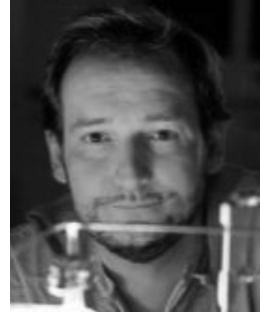

Emanuele Pelucchi received the $\mathrm{Ph} . \mathrm{D}$. degree in 1997, in the field of surface science and the M.B.E. degree from TASC National Laboratory, Italy. His research interests include MBE of II-VI materials, photoemission spectroscopy applied to interface physics and metal-semiconductor barriers. He moved to Lausanne in 2001 as a Postdoctoral Researcher, where he was involved in the research of MOVPE of site controlled III-V nanostructures, on both V-groove quantum wires and pyramidal QDs.

In May 2006, he received the Science Foundation Ireland Principal Investigator Grant and moved to the Tyndall Research Institute in January 2007 where he set up a new research group in the field of III-V epitaxy for device integration and semiconductor quantum dots. He is currently the Head of group at Tyndall-UCC and jointly a Senior Research Fellow of the University Department of Physics. He has coauthored more than 100 publications and is currently supervising three Ph.D. students and two postdoctoral researchers.

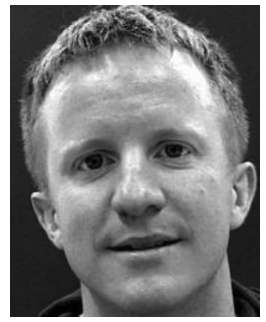

Kevin Thomas was born in the U.K., in 1967. He received the B.Sc. (Hons.) degree in chemistry in 1989 and the Ph.D. degree in surface chemistry and electron spectroscopy for chemical analysis in 1994, both from the University of Cardiff, Cardiff, U.K. From 1996 to 1998, he was a MOVPE Production and Development Engineer with International Quantum Epitaxy, Cardiff. Since 1998, he has been a Research Scientist and the Laboratory Manager in MOVPE and III-V Material Characterization at Tyndall National Institute, University College Cork, Cork, Ireland.

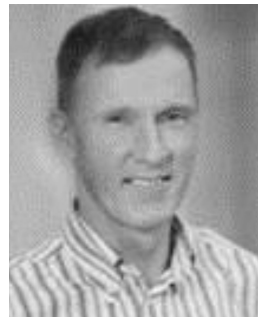

Brian Corbett received the B.A. Mod. degree in experimental physics and mathematics and the M.Sc. degree in theoretical physics from Trinity College, Dublin, Ireland. Since 1991, he has been a Scientist at the Tyndall National Institute, University College Cork, Cork, Ireland, researching the physics and technology of III-V-based light-emitting semiconductors. His research interests include design, fabrication, and characterization of photonic devices with special emphasis on the use of advanced structuring technologies to add additional function to the device. Examples range from facetless lasers using reflective sites, high-brightness unstable resonators, surface-emitting lasers with gratings and three-dimensional shaping to collimate light from LEDs. The devices being studied are based on InP, GaAs, $\mathrm{GaN}, \mathrm{Si}$, and $\mathrm{Ge}$ materials. 